\title{
Analytical and empirical evaluation of the impact of Gaussian noise on the modulations employed by Bluetooth Enhanced Data Rates
}

\author{
José Rafael Luque*, María José Morón and Eduardo Casilari
}

\begin{abstract}
Bluetooth (BT) is a leading technology for the deployment of wireless Personal Area Networks and Body Area Networks. Versions 2.0 and 2.1 of the standard, which are massively implemented in commercial devices, improve the throughput of the BT technology by enabling the so-called Enhanced Data Rates (EDR). EDRs are achieved by utilizing new modulation techniques ( $\pi / 4-D Q P S K$ and 8-DPSK), apart from the typical Gaussian Frequency Shift Keying modulation supported by previous versions of BT. This manuscript presents and validates a model to characterize the impact of white noise on the performance of these modulations. The validation is systematically accomplished in a testbed with actual BT interfaces and a calibrated white noise generator.
\end{abstract}

Keywords: Bluetooth, bit error rate, modulation, white noise

\section{Introduction}

Bluetooth (BT) is one of the most broadly adopted technologies for the development of short range and lowpower networking applications in Personal Area Networks and Body Area Networks.

Initially conceived for cable replacement, BT has expanded into a wide variety of electronic devices ranging from handheld products (laptops, electronic pads, smartphones, pen tablets) to automobile electronics, portable digital music players, peripherals, headsets, wireless sensor and gaming consoles such as Sony PlayStation 3 or Nintendo Wii. This progress of BTenabled hardware promoted the delivery of new versions of the standard aimed at satisfying the growing bandwidth requirements of the networking applications.

The first increase of the basic BT transmission rate was introduced by BT $2.0+E D R$ standard [1], which is thoroughly compatible with previous versions 1.2 and 1.1 as it incorporates all their functional characteristics. Specifically, its major innovation resides in the optional utilization of two new Differential Phase Shift Keying (DPSK)-based modulation schemes to enable a faster data transfer through the so-called Enhanced Data Rates (EDR). These novel schemes ( $\pi / 4-D Q P S K$ and 8-DPSK)

\footnotetext{
* Correspondence: rluque@uma.es

Departamento de Tecnología Electrónica, University of Málaga, Málaga, Spain
}

are employed to transmit the BT packet payload at a bit rate (2 or $3 \mathrm{Mbps}$, respectively) higher than the $1 \mathrm{Mbps}$ basic speed of BT 1.1 and 1.2, which is achieved with a Gaussian Frequency Shift Keying (GFSK) modulation. These EDR modulations are also employed in the 2.1 version of BT [2].

BT physical layer works on the Industrial, Scientific and Medical (ISM) 2.4 GHz unlicensed band. As a consequence, $\mathrm{BT}$ radio transmissions are vulnerable to the interferences of other BT devices as well as other technologies operating in the same ISM band. Because of the wide expansion of some of these technologies (especially BT and 802.11), most practical scenarios of BT-capable applications will be deployed under a high environmental radio noise. The effects of the noise level on the bit errors experimented by the BT signal strongly depend on the employed modulation scheme. This article empirically evaluates the impact of Gaussian noise on the Bit Error Rate (BER) suffered by real BT transmissions. The study permits to assess the accuracy of existing analytical models that relates the BER to the Signal-to-Noise Ratio (SNR) for the considered modulations.

The rest of the article is organized as follows. Section 2 reviews and classifies the literature related to the modeling and performance evaluation of the different modulations (GFSK, $\pi / 4$-DQPSK and 8-DPSK) contemplated 
by the BT 2.x+EDR (2.0 and 2.1) specifications. Based on existing studies, Section 3 analytically characterizes the BER as a function of the SNR for these different modulations. Section 4 evaluates the validity of these analytical models in an experimental testbed with actual interfaces provided with a commercial BT firmware. Finally, Section 5 summarizes the main conclusions of the article.

\section{Related study}

There is a vast literature on the characterization of the performance of the existing modulation techniques for RF communications. The relationship between the BER experienced by the transmissions and the SNR has extensively been studied. General analytical models for the GFSK [3], $\pi / 4-D Q P S K ~[4-11]$ and 8-DPSK [12-14] modulations have been proposed.

Some of these general models are particularized for characteristics of the transceivers which are implemented by the BT standard. For example, authors of [15] present an analytical study of the GFSK modulation with noncoherent detection. The study focuses on systems that employ frequency hopping as the spread spectrum technique (which is the case of BT). The analysis, which is performed for Rayleigh-type channels, results in a model that relates the BER to the SNR ratio and the modulation index. In addition, a method to improve the performance of frequency-hopping systems is proposed.

The study in [16] describes the theoretical design of a GFSK transceiver with carrier frequency offset correction. The design introduces a mechanism to simplify the implementation of a multi-symbol receiver. In order to reduce the $B E R$, the proposed mechanism utilizes an algorithm that compensates the carrier frequency offset. The study shows some results when this proposal is applied to a BT receiver.

After examining the existing channel models in the literature for different propagation conditions, the study in [17] proposes a discrete channel model (based on Markov chains) to evaluate BT piconets by simulations.

A new scheme for a digital GFSK transceiver is proposed in [18]. The main novelty lies in the method employed to estimate the carrier frequency. The model for the computation of the BER, which is validated by means of simulations, assumes an additive white Gaussian noise (AWGN) channel. A similar carrier synchronization mechanism is presented in [19]. In that paper, least squares (LS) and maximum likelihood (ML) techniques are employed for decision-aided carrier recovery in a GFSK receiver. By analyzing the estimation error variance, the LS/ML carrier frequency offset estimation method is shown to outperform the traditional method using the discrete-time Fourier transform. In addition, the proposed scheme (which is not implemented and whose computational complexity is not evaluated) is compared by simulations with the DFTbased method in terms of the obtained BER. Noise is again characterized through an AWGN channel.

On the other hand, there are works in the literature specifically dealing with the characterization of the impact of the noise on BT transmissions. For example, authors of [20] analyze the performance of 1.1 version of BT technology in Gaussian and Rayleigh-type channels. The study offers analytical expressions (with some mathematical mistakes) to compute the transmission rate that can be achieved by the different $\mathrm{BT}$ packet types as a function of the SNR. For this purpose, the Packet Error Rate is calculated from the BER obtained with a GFSK modulation. The article discusses the utility of the different packet types depending on the considered channel type (Raleigh or AWGN).

An RF simulation system for the development of commercial BT transceiver was initially introduced in [21]. The emulation of the channel permits to combine an AWGN and the existence of interfering signals.

The article in [22] designs a policy to select the BT packet type depending on the estimation of the channel SNR. The authors of that article suggest the use of new modulations (which are not considered by BT 2.x+EDR) and new algorithms to compute the SNR. However, the feasibility of the implementations of these algorithms at the LM (Link Manager) layer of actual BT host controller is not discussed.

Author in [23] studies the behavior of BT transmissions in Nakagami-type channels. Results are based on simulations that estimate the BER as a function of the existing SNR. Analyses are conducted for different values of the fading parameter of the Nakagami distribution (as well as for an AWGN channel).

The study in [24] extends the model presented in [20] (conceived for the modulation employed by BT 1.1) to the EDR modes. As in [20], the benefits of using the new packet types under an AWGN channel are discussed. In this sense, a strategy for selecting the packet type as a function of the SNR is described. The article suggests deploying this selection at the L2CAP BT layer. However, it does not indicate how this decision must be executed at the Baseband layer.

In [25], the authors provide a Matlab simulation model for the physical layer of a transceiver implementing BT 2.0+EDR. The BER obtained with this model for different noise levels is contrasted with theoretical models which also assume an AWGN channel.

The study in [26] investigates the performance of Wireless Application Protocol data applications over the BT protocol stack. The performance of the communications is evaluated by assuming again that bit errors in a simulated BT piconet are induced by an AWGN channel. 
Based on Markov chains, Zanella [27] presents a mathematical analysis of the BT 2.0+EDR transmissions in fading channels. In [28], Zanella performs a theoretical characterization of the bit error probability for BT 2.0 transmissions under both AGWN and Rice fading channels.

Table 1 lists the abovementioned studies about the characterization of the different modulations employed by BT. The table also includes the references to different articles dealing with the optimization and implementation of BT demodulators for GFSK, $\pi / 4$-DQPSK, and 8-DPSK modulations.

In some of these previous studies, the proposed analytical models are not validated. In other cases, the evaluation is just carried out by means of simulations. To the best of the authors' knowledge, no study offers a validation of the models with actual BT devices. The goal of this study is to fill this gap by combining the analytical modeling with the empirical evaluation of the influence of noise on the errors experienced by BT transmissions.

\section{Characterization of the modulations employed by BT 2.x+EDR in an AWGN channel}

The goal is to analytically relate the BER of the BT transmissions to the existing SNR for GFSK, $\pi / 4$ DQPSK, and 8-DPSK modulations. In this sense, as commented in the previous state-of-the-art, the characterization of the transmission channel as an AWGN channel has widely been considered by the literature for the analysis of the performance of BT connections and even for the design of $\mathrm{BT}$ radio transceivers. In studies on BT technology, several reasons have been argued to justify the use of this channel characterization:

- BT communications are usually deployed in static (indoor) office scenarios. Thus, the transmission model can presume that short-term (fast) fading has a minor impact on BT performance.

- The restrictions on the transmission power of BT devices impose low-range communications where Line-of-Sight from the receiver to the transmitter normally exists.

- In this sense, indoor environments are typically characterized by the presence of other interfering technologies (such as Wi-Fi) operating in the same BT 2.4 GHz ISM band. These interference sources (with different spectral properties) can reasonably be approximated by a noise with a constant spectral density.

An AWGN channel adds a flat power white noise to the whole considered signal bandwidth. As the effect of an AWGN channel is the addition of a noise with constant energy in the whole signal bandwidth, the SNR ratio can be computed [29] as

$$
\mathrm{SNR}=\frac{P_{\mathrm{r}}}{N_{\mathrm{o}} \cdot B}
$$

where $P_{r}$ is the power at the receptor, expressed (in W), $N_{\mathrm{o}} / 2$ is the noise added by the channel, defined in terms of the Power Spectral Density (in W/Hz), and $2 B$ is the considered bandwidth (in $\mathrm{Hz}$ ) of the signal at the reception.

In order to compare the effect of noise on the different digital modulations employed by BT 2.x+EDR, we characterize the SNR as a function of the energy transmitted per bit or per symbol

$$
\mathrm{SNR}=\frac{P_{\mathrm{r}}}{N_{0} \cdot B}=\frac{E_{\mathrm{S}}}{N_{0} \cdot B \cdot T_{\mathrm{S}}}=\frac{E_{\mathrm{b}}}{N_{0} \cdot B \cdot T_{\mathrm{b}}}
$$

where $E_{\mathrm{s}}$ and $T_{\mathrm{s}}$ represent the energy per transmitted symbol (expressed in $\mathrm{W}$ ) and the symbol transmission period, respectively. $E_{\mathrm{b}}$ and $T_{\mathrm{b}}$ denote the energy per transmitted bit (in $\mathrm{W}$ ) and the bit transmission period.

As $B, T_{\mathrm{s}}$, and $T_{\mathrm{b}}$ are known constant values in $\mathrm{BT}$, the SNR can directly be referred to the SNR ratios per symbol $\left(\gamma_{\mathrm{s}}\right)$ and bit $\left(\gamma_{\mathrm{b}}\right)$, defined as [29]

$$
\begin{aligned}
& \gamma_{\mathrm{s}}=\frac{E_{\mathrm{s}}}{N_{0}} \\
& \gamma_{\mathrm{b}}=\frac{E_{\mathrm{b}}}{N_{0}}
\end{aligned}
$$

\begin{tabular}{|c|c|c|c|}
\hline \multirow[t]{2}{*}{ Topic } & \multicolumn{3}{|c|}{ Modulation scheme } \\
\hline & GFSK & $\pi / 4-D Q P S K$ & 8-DPSK \\
\hline Generic analytical models & [3] & {$[4-11]$} & {$[12-14]$} \\
\hline Generic models particularized for BT characteristics & [15-19] & & \\
\hline Specific analytical studies on BT & {$[20,21,23,26,28]$} & {$[24,25,28]$} & \\
\hline Design and implementation of BT demodulators & {$[40-47]$} & {$[48]$} & \\
\hline
\end{tabular}

The relationship between these two parameters can be computed as

Table 1 Taxonomy of the scientific literature on the relationship BER-SNR for BT modulations

A taxonomy of the scientific literature on the relationship between the BER rate and the SNR for the different modulations employed by BT 


$$
\gamma_{\mathrm{b}}=\frac{\gamma_{\mathrm{s}}}{\log _{2} M}
$$

where $M$ indicates the number of symbols of the modulation constellation.

The next step is to determine the BER, defined as the bit error probability, $P_{\mathrm{b}}$, as a function of $\gamma_{\mathrm{b}}$, for all the modulations specified by BT 2.x+EDR. For the case of multi-level symbol constellations, $P_{\mathrm{b}}$ can be obtained from the probability of symbol error $\left(P_{\mathrm{s}}\right)$ and the number $(M)$ of existing constellation symbols. Assuming that bit errors are uncorrelated, for low values of $P_{\mathrm{s}}$ (e.g., below 0.1) $P_{\mathrm{b}}$ can be reasonably approximated [29] as

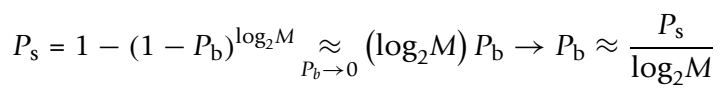

\subsection{GFSK modulation}

GFSK scheme can be assimilated to an FSK modulation. To determine the bit error probability, we have to take into account that BT was conceived as a low-cost technology. At the expense of a lower performance, noncoherent detection is preferred to simplify the hardware architecture of the demodulator. When this type of detection is utilized, the bit error probability can be computed [30] as

$$
P_{\mathrm{b}}=\frac{1}{2} e^{\left(-\frac{\gamma_{\mathrm{b}}}{2}\right)}
$$

The previous expression in Equation (7) describes a lower bound for $P_{\mathrm{b}}$ which assumes an orthogonal modulation scheme with a modulation index $h$ of 0.5 . However, BT specification imposes the index $h$ to have a value in the interval [0.28-0.35], lower than 0.5. Under this non-orthogonal modulation, $P_{\mathrm{b}}$ can be estimated [31] from

$$
P_{\mathrm{b}}=Q_{1}(\alpha, \beta)-\frac{1}{2} e^{\left(-\frac{\alpha^{2}+\beta^{2}}{2}\right)} I_{0}(\alpha, \beta)
$$

where $Q_{1}(\alpha, \beta)$ represents the Marcum Q-function, which is defined as

$$
Q_{1}(\alpha, \beta)=\int_{\beta}^{\infty} x e^{\left(-\frac{x^{2}+\alpha^{2}}{2}\right)} I_{0}(\alpha x) d x=e^{\left(-\frac{\alpha^{2}+\beta^{2}}{2}\right)} \cdot \sum_{n=0}^{\infty}\left(\frac{\alpha}{\beta}\right)^{n} I_{n}(\alpha \beta) ; \quad(\beta>\alpha>0)
$$

where $I_{n}(x)$ expresses the $n$ th-order modified Bessel function. In Equation (8), $\alpha$ and $\beta$ are two parameters that can be calculated from $\gamma_{\mathrm{b}}[31]$

$$
\alpha=\sqrt{\frac{\gamma_{\mathrm{b}}}{2}\left(1-\sqrt{1-\rho^{2}}\right)}
$$

$$
\beta=\sqrt{\frac{\gamma_{\mathrm{b}}}{2}\left(1+\sqrt{1-\rho^{2}}\right)}
$$

In the previous expressions, the term $\rho$ is the so-called correlation coefficient, which can be calculated from the modulation index $h$

$$
\rho=\frac{\sin (2 \pi h)}{2 \pi h}
$$

Therefore, to calculate the bit error probability, $P_{\mathrm{b}}$, for transmissions with the basic rate of $\mathrm{BT}$, we just have to consider that the modulation index $h$ is in the range [0.28-0.35]. In any case, this computation neglects the effect of the Inter-Symbol Interference (ISI) which is introduced by the Gaussian filter implemented by GFSK.

\section{2. $\pi / 4-D Q P S K$ modulation}

As it refers to the performance of the radio receptor, $\pi /$ 4-DQPSK (Differential Quaternary PSK) modulation has an identical behavior to DQPSK (4-DPSK) for an ideal lineal AWGN channel [32]. Thus, for $\pi / 4$-DQPSK, the symbol error probability, $P_{\mathrm{s}}$, can be approximated [32] as

$$
P_{\mathrm{s}}=\frac{\sin \frac{\pi}{M}}{2 \pi} \int_{-\pi / 2}^{\pi / 2} \frac{\exp \left(\gamma_{\mathrm{s}} \cdot\left(1-\cos \frac{\pi}{M} \cdot \cos \theta\right)\right)}{\left(1-\cos \frac{\pi}{M} \cdot \cos \theta\right)} d \theta
$$

The $\pi / 4-D Q P S K$ constellation defines four different symbols $(M=4)$, so that 2 bits are codified per symbol. On the other hand, once the value of $M$ is known, $P_{\mathrm{b}}$ and $\gamma_{\mathrm{s}}$ can be easily derived from $P_{\mathrm{s}}$ and $\gamma_{\mathrm{b}}$ by applying Equations (6) and (5), respectively. So, we have that the expression for the calculus of $P_{\mathrm{b}}$ under $\pi / 4-\mathrm{DQPSK}$ is

$$
P_{\mathrm{b}}=\frac{1}{\log _{2} M} \cdot \frac{\sin \frac{\pi}{M}}{2 \pi} \int_{-\pi / 2}^{\pi / 2} \frac{\exp \left(\left(\gamma_{\mathrm{b}} \cdot \log _{2} M\right) \cdot\left(1-\cos \frac{\pi}{M} \cdot \cos \theta\right)\right)}{\left(1-\cos \frac{\pi}{M} \cdot \cos \theta\right)} d \theta
$$

\subsection{8-DPSK modulation}

In this case, Equation (14) also permits to calculate the bit error probability, $P_{\mathrm{b}}$, if $M$ is set to 8 (as 8 -DPSK codifies $\log _{2} 8=3$ bits per symbol). However, as it is remarked in [31], the exact computation of this formula for $P_{\mathrm{b}}$ under $M$-DPSK becomes very complex for values of $M$ higher than 2. Authors of [32] provide an upper bound for the value of $P_{\mathrm{s}}$ which can be computed as

$$
P_{\mathrm{s}} \leq 2.06 \cdot \sqrt{\frac{1+\cos \frac{\pi}{M}}{2 \cdot \cos \frac{\pi}{M}}} \cdot Q\left(\sqrt{\gamma_{\mathrm{s}}\left(1-\cos \frac{\pi}{M}\right)}\right)
$$


From this expression and Equation (6), $P_{\mathrm{b}}$ can easily be derived.

\section{Empirical evaluation of the model}

\subsection{Description of the employed testbed}

In order to empirically evaluate the accuracy of the above-presented theoretical models as well as the impact of the noise on the bit errors experienced by actual BT transmissions, we deployed the testbed shown in Figure 1. Aiming at emulating the Gaussian channel in a fully controlled environment, we construct a scenario where the transmissions between two BT devices are uniquely affected by two externally tunable parameters: an attenuation factor and an additive Gaussian white noise. For this purpose, the testbed introduces a signal attenuator and a noise generator between the two BT interfaces (acting as the BT master and slave, respectively). To avoid any external interference, all the connections between the utilized devices are performed through coaxial cables with SMA connectors. The attenuation introduced by the cables and the connectors is proved to be less than $0.5 \mathrm{~dB}$.

The following sections describe the characteristics of these elements of the measurement system, which has been depicted in Figure 2.

\subsubsection{BT devices}

The deployed testbed utilizes two BT development boards from Bluegiga vendor [33]. In particular, these boards are those included in the EKWT11-E development kit [34], which also incorporates a Serial Peripheral Interface (SPI) programming interface. Each board integrates a WT11-E BT module with the Bluecore-4 chipset from CSR (the most popular manufacturer of BT technology). These class-1 modules incorporate a flash memory that permits to modify the module firmware. Thus, by using the SPI interface, the original firmware from Bluegiga in the modules was updated with a
CSR firmware version fully compatible with the BT 2.0 +EDR specification. More precisely this version employs:

- HCI Version: $2.0(0 \times 3)$ and revision: 0xafa

- LM Version: 2.0 (0x3) and subversion: 0xafa

\subsubsection{Signal attenuator}

In order to simulate the attenuation introduced by the radio propagation in the $2.4-\mathrm{GHz}$ ISM band between the transmitter and the receiver, we utilize two adjustable microwave attenuators (the 8494B [35] and 8496B [36] models from Agilent). These manual step attenuators are able to operate from 0 to $18 \mathrm{GHz}$. The $8494 \mathrm{~B}$ model provides a calibrated attenuation level from 0 to $11 \mathrm{~dB}$ with an attenuation step of $1 \mathrm{~dB}$. Similarly, the range and attenuation step of the $8496 \mathrm{~B}$ model are [0-110 dB] and $10 \mathrm{~dB}$, respectively. As depicted in Figure 1, the two devices are coupled in series and connected with the SMA antenna connector of the transmitting BT board. Thus, a global adjustable signal attenuation of up to 121 $\mathrm{dB}$ with a resolution of $1 \mathrm{~dB}$ can be achieved.

\subsubsection{Noise generator}

The White Gaussian noise is introduced by a NoiseCom NC6124A Noise Generator by [37]. This model injects a white noise with a power level of $-10 \mathrm{dBm}$ in the $2-4$ $\mathrm{GHz}$ band. The equipment integrates an attenuator which can reduce the noise power up to $10 \mathrm{~dB}$ with 1 $\mathrm{dB}$ steps. The noise is added to the BT signal by a combiner also included in the generator.

\subsection{Measurements of the SNR and the BER at the receptor}

BER has been measured using the test capabilities of the RF layer implemented in the utilized BT modules. Under the test mode [1,2], the RF subsystem module is configured to estimate a set of quality and performance parameters (including the BER) defined by the BT specification for the

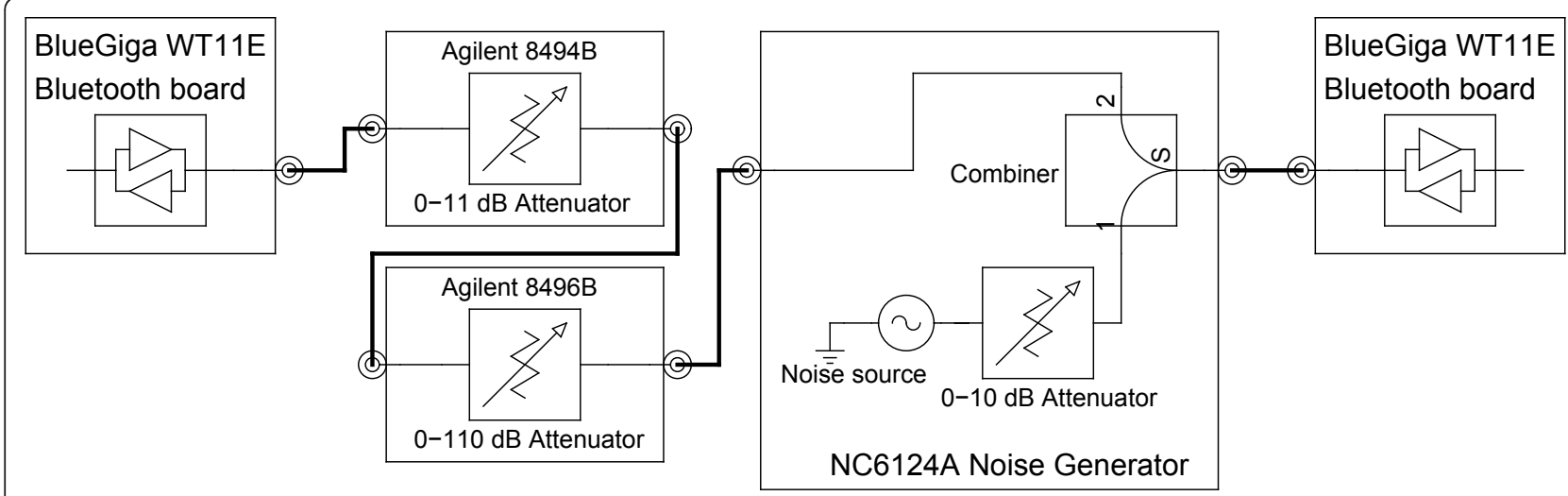

Figure 1 Connection diagram of the testbed 


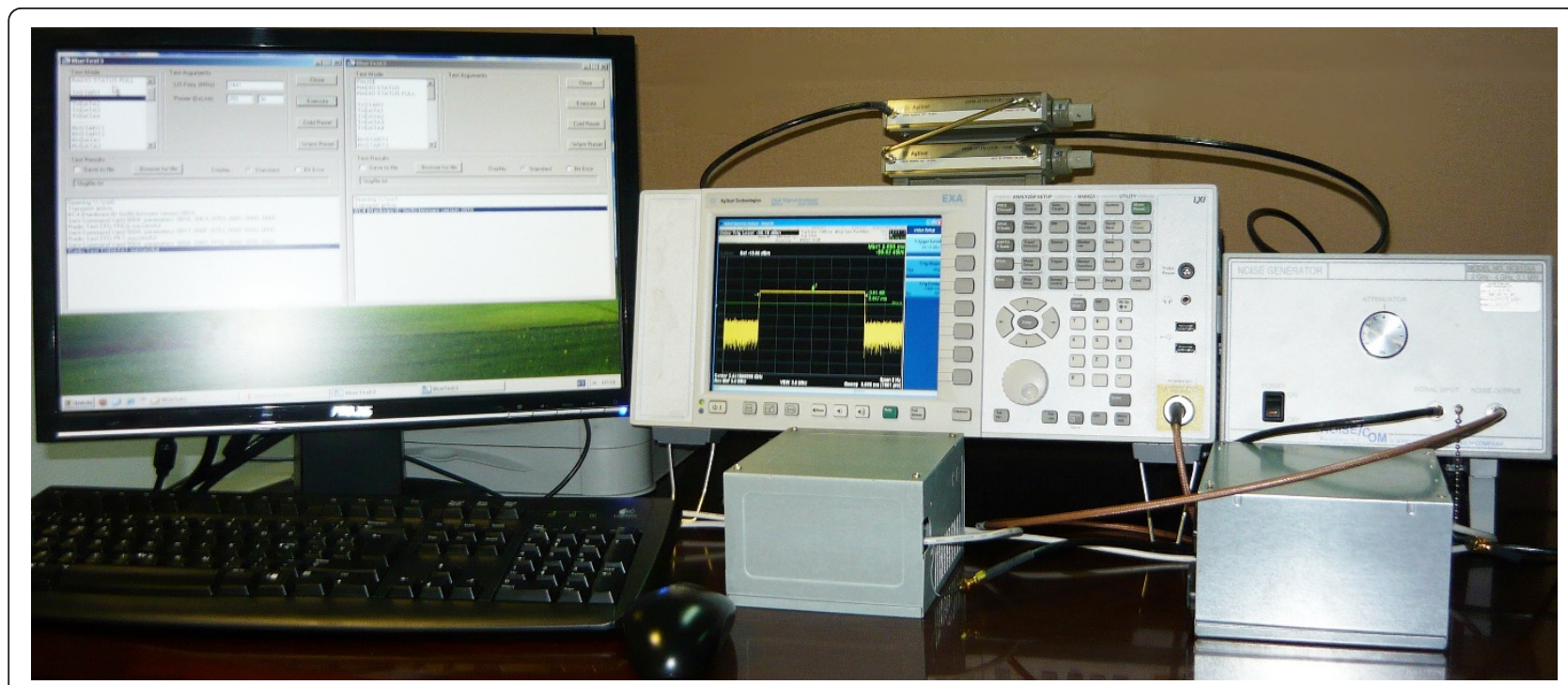

Figure 2 Employed testbed for the measurements.

RF layer. In particular, we have employed BlueTest, an application provided by CSR which allows to set up this test mode in modules incorporating CSR chipsets.

The programmed test mode in the Bluegiga devices estimates the BER after the emission of a series of 339byte packets. Packets are emitted at a rate of 200 packets per second (with a constant time of $5 \mathrm{~ms}$ between consecutive packets). These probe packets contain a predefined Pseudo-random bit sequence (specified in [38] as PRBS9 payload pattern) so that errors can easily be detected at the receptor. This PRBS9 sequence (which is generated from a feedback 9-stage shift register) is conceived to run performance tests on digital transmission equipments. The test program actually calculates the residual BER, i.e., after decoding and applying Forward Error Correction (FEC) to the received data. Thus, for the tests, DH5type packets are employed as the data in the payload of these 5-slot packets are not FEC encoded.

Besides, aiming at estimating the actual SNR, an N9010A spectrum analyzer from Agilent Technologies [39] has been utilized to measure the actual power densities of the noise and the signal at the BT receptor.

BT technology optionally enables the use of an adaptive transmission power control system. Based on the feedback messages describing the receiver signal strength, this control allows the emitter to regulate the transmission power so that the power at the receptor always keeps in a preset range (the "golden receive power range"). In our experiments, in order to ensure that the emitting power does not vary during the transmissions, the signal attenuation is set to a certain constant value $(45 \mathrm{~dB})$. This attenuation guarantees that the signal power at the reception remains within a range $([-40,-60]$
$\mathrm{dBm}$ for the BlueCore- 4 chipset) where the receptor disables the automatic gain control and consequently no feedback to the transmitter is executed to modify the transmitting power. With this value of the signal attenuation, from the power measurements obtained with the spectrum analyzer, we can deduce that

- The noise density level in the input of the receptor (if no attenuation is selected in the generator) is $-109.1 \mathrm{dBm} / \mathrm{Hz}$, with a deviation of less than $1 \mathrm{~dB}$ for the whole operation bandwidth.

- The signal density level at the receptor varies from -101.7 to $-102.6 \mathrm{dBm} / \mathrm{Hz}$, depending on the $\mathrm{RF}$ channel utilized by BT. In our analysis, we consider the average value of $-102.15 \mathrm{dBm} / \mathrm{Hz}$.

From these two values of the received power density of the noise and the signal, we have that the SNR at the reception point is $6.95 \mathrm{~dB}$ for zero noise attenuation. By increasing the adjustable attenuation in the noise generator, we can evaluate the BER for different SNR scenarios.

\subsection{Analysis of the obtained results}

In this section, we evaluate the accuracy of the analytical models presented in Section 3 to fit the BER that is obtained in the empirical testbed for different values of the SNR.

The measured values of the BER, for a constant signal attenuation $(45 \mathrm{~dB})$ and different levels of noise attenuation, are tabulated in Table 2 for the three studied modulations (GFSK, $\pi / 4$-DQPSK, and 8-DPSK). Note that the table does not show some results obtained with the 
$\pi / 4$-DQPSK modulation and high noise attenuation, due to the difficulty of measuring a stable value of the BER when this parameter is very low. Similarly, the results for the 8-DPSK modulation are not represented when noise induces so many losses in the BT connections that they become unviable.

Figures 3, 4, and 5 compare these empirical results with those computed with the analytical models when the same values of the SNR ratio (expressed in terms of $\left.\gamma_{\mathrm{b}}\right)$ are utilized as the input variable of the corresponding equations. In particular, Figure 3 depicts the results obtained for the GFSK modulation employed by the BT basic rate of 1 -Mbps. The graphs include the values of the BER measured in the testbed when the basic mode is selected in the transmitter, as well the BER calculated by the analytical model (defined by Equation 8 ) for three different modulation indexes $(h): h=0.28, h=$ 0.30 , and $h=0.35$. Results show that for high values of the SNR (i.e., those above $14 \mathrm{~dB}$ ), the measured vales are clearly higher than those computed analytically. This divergence could be explained by the fact that the analytical model does not take into consideration the secondorder effects, such as the ISI introduced by the Gaussian filter employed by the GFSK modulator. Unfortunately, it is not possible to analytically characterize the ISI without taking into consideration the utilized demodulation technique and its specific hardware implementation in the BT interfaces. The particular characteristics of these elements are unknown in the actual testbed, as the vendors do not offer a detailed description of their modulator/demodulator modules. Nevertheless, from the results, it is inferred that ISI only (slightly) increases the BER in scenarios with low noise (and low BER). In any case, the impact of this low BER on the actual performance of the BT transmissions is negligible. In fact,

\begin{tabular}{|c|c|c|c|}
\hline \multirow[t]{2}{*}{ Noise attenuation (dB) } & \multicolumn{3}{|c|}{ Measured BER } \\
\hline & GFSK & $\pi / 4-D Q P S K$ & 8-DPSK \\
\hline 0 & $4.24 \times 10^{-2}$ & $4.84 \times 10^{-2}$ & - \\
\hline 1 & $2.67 \times 10^{-2}$ & $3.14 \times 10^{-2}$ & - \\
\hline 2 & $1.49 \times 10^{-2}$ & $1.50 \times 10^{-2}$ & - \\
\hline 3 & $9.22 \times 10^{-3}$ & $8.00 \times 10^{-3}$ & $8.70 \times 10^{-2}$ \\
\hline 4 & $5.00 \times 10^{-3}$ & $4.28 \times 10^{-3}$ & $6.70 \times 10^{-2}$ \\
\hline 5 & $2.3 \times 10^{-3}$ & $1.53 \times 10^{-3}$ & $4.20 \times 10^{-2}$ \\
\hline 6 & $1.1 \times 10^{-3}$ & $6.90 \times 10^{-4}$ & $3.10 \times 10^{-2}$ \\
\hline 7 & $4.8 \times 10^{-4}$ & $1.84 \times 10^{-4}$ & $2.14 \times 10^{-2}$ \\
\hline 8 & $1.3 \times 10^{-4}$ & $6.10 \times 10^{-5}$ & $1.24 \times 10^{-2}$ \\
\hline 9 & $5.7 \times 10^{-5}$ & - & $8.20 \times 10^{-3}$ \\
\hline 10 & $1.7 \times 10^{-5}$ & - & $4.70 \times 10^{-3}$ \\
\hline
\end{tabular}

Results of the BER measurements obtained for GFSK, $\pi / 4-D Q P S K$, and 8-DPSK modulations for a $45-\mathrm{dB}$ signal attenuation for a value of the SNR above $14.95 \mathrm{~dB}$ (obtained in the testbed with a noise attenuation of $8 \mathrm{~dB}$ ), the erroneous packets and, consequently, the increase of the delay provoked by packet retransmissions are practically undetectable. To show this, Figure 6 represents the mean delay experienced by the data at the user layer when they are transmitted by a BT connection using the Serial Port Profile. The figure compares this mean delay (for different data sizes) obtained in the testbed in ideal conditions (when no noise is added) and for an SNR of $14.95 \mathrm{~dB}$. The graphs (which are indistinguishable) clearly indicate that an SNR above a certain level has no practical consequences on the behavior of BT transmissions. Thus, an exact characterization of the relationship between the BER and the SNR when the SNR is above this level could not be necessary.

Conversely, for SNR values lower than $10 \mathrm{~dB}$ (and high values of the BER), the measured BER is always slightly below the analytical estimations, even for the highest modulation index $(h=0.35)$. This can be justified by the fact that the estimation of the empirical BER only computes the errors existing in packets that have properly been received (and corrected). Therefore, it does not consider the errors included in those packets that have been lost because of the high noise level. Nevertheless, this underestimation of the BER can be again disregarded as, in an actual scenario, such a magnitude of the BER would provoke the loss of the BT connection. Consequently, the transmission conditions render BT transmission virtually infeasible for this range of the SNR (in fact, performed tests show that the analysis of the packet delay cannot be properly executed because of the high probability of a connection loss).

The graphs in Figures 4 and 5 show the results obtained when the experiments are repeated with $\pi / 4$ DQPSK and 8-DPSK modulations, respectively. In the first case, we use the theoretical model defined by Equation (14) for $\pi / 4$-DQPSK modulation, where $M$ is given a value of $4(M=4)$. For 8 -DPSK modulation (which uses a constellation of eight symbols), Equation (15) is employed with $M=8$.

Under 8-DPSK modulation, transmissions with a BER over 0.1 are not feasible (due to the excessive number of packet retransmissions and the losses of connections that errors provoke). Figure 5 shows that the analytical model performs an accurate adjustment of the BER in the range [0.1, 0.01].

For 4-DQPSK, which is a more robust modulation, tests with a BER greater than 0.1 are possible. In this case, Figure 4 illustrates that the model fits the experimental BER up to values of 0.001 more adequately.

The mismatch of the models for the scenarios with low noise (high values of $\gamma_{\mathrm{b}}$ ) can be explained again by the effects of the ISI as well as by limitations and 


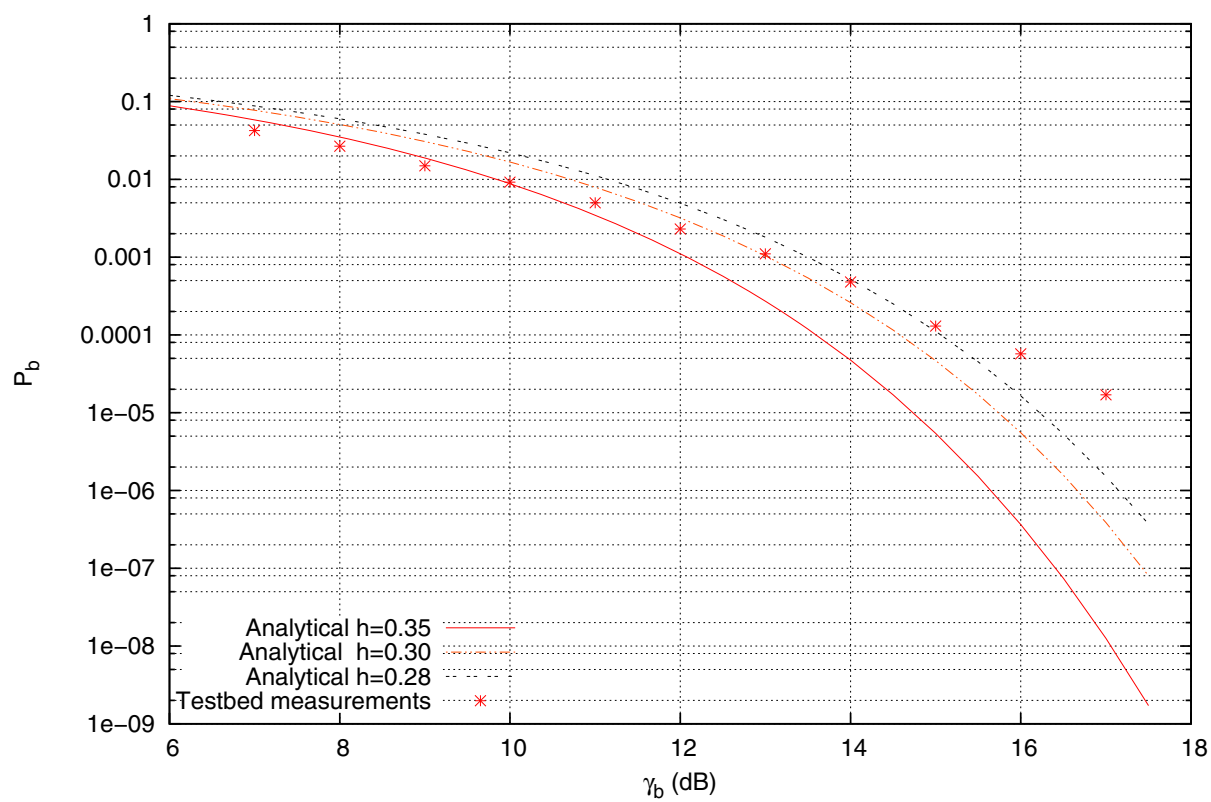

Figure 3 Bit error probability $\left(P_{\mathrm{b}}\right)$ as a function of the SNR per bit $\left(\gamma_{\mathrm{b}}\right)$ for the basic rate (GFSK modulation). Comparison between the analytical approximation and the empirical measurements.

inaccuracies of the hardware of the phase detector in the demodulator. The non-idealities of the phase detector may degrade the performance of the demodulator. Certainly, the theoretical model computes an upper bound of the BER but assuming ideal modulators/demodulators. That is the reason why the model (for 4-DQPSK and 8-DPSK modulations) outperforms the measurements.

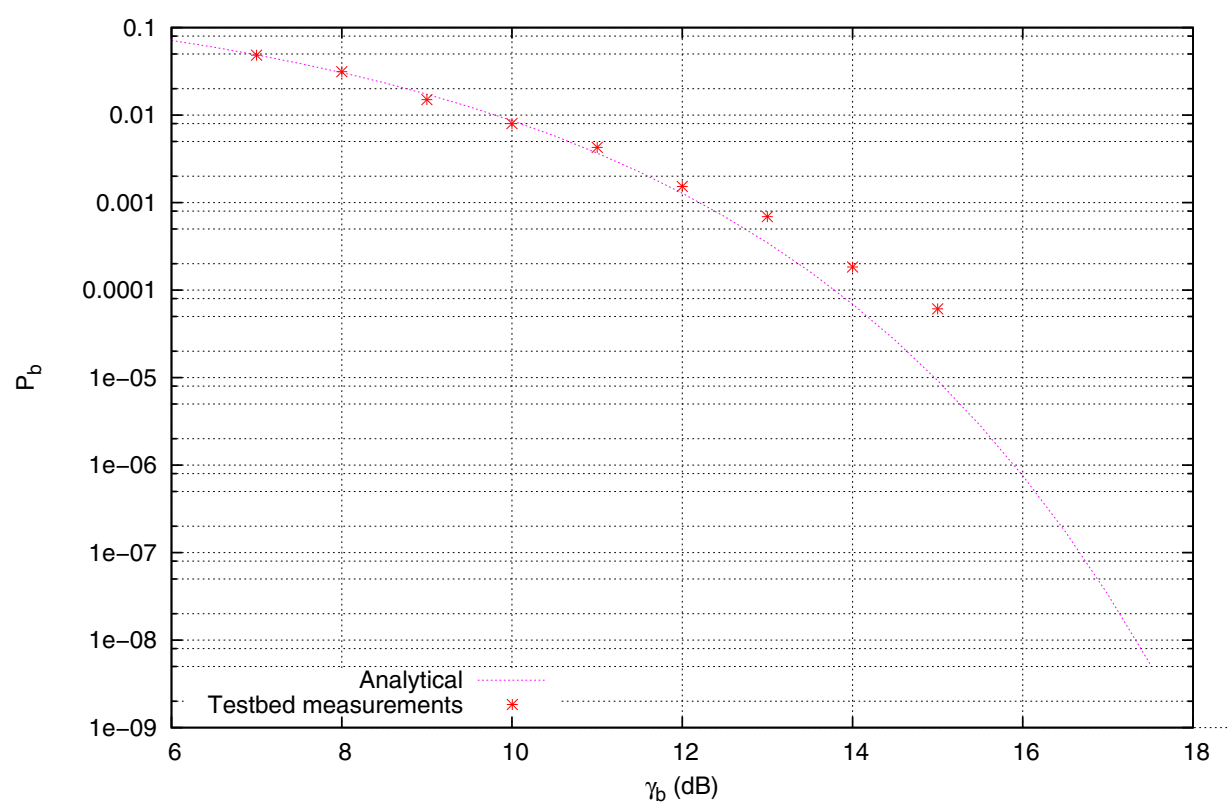

Figure 4 Bit error probability $\left(P_{b}\right)$ as a function of the SNR per bit $\left(\gamma_{b}\right)$ for the 2-Mbps rate mode ( $\pi / 4-D Q P S K$ modulation). Comparison between the analytical approximation and the empirical measurements. 


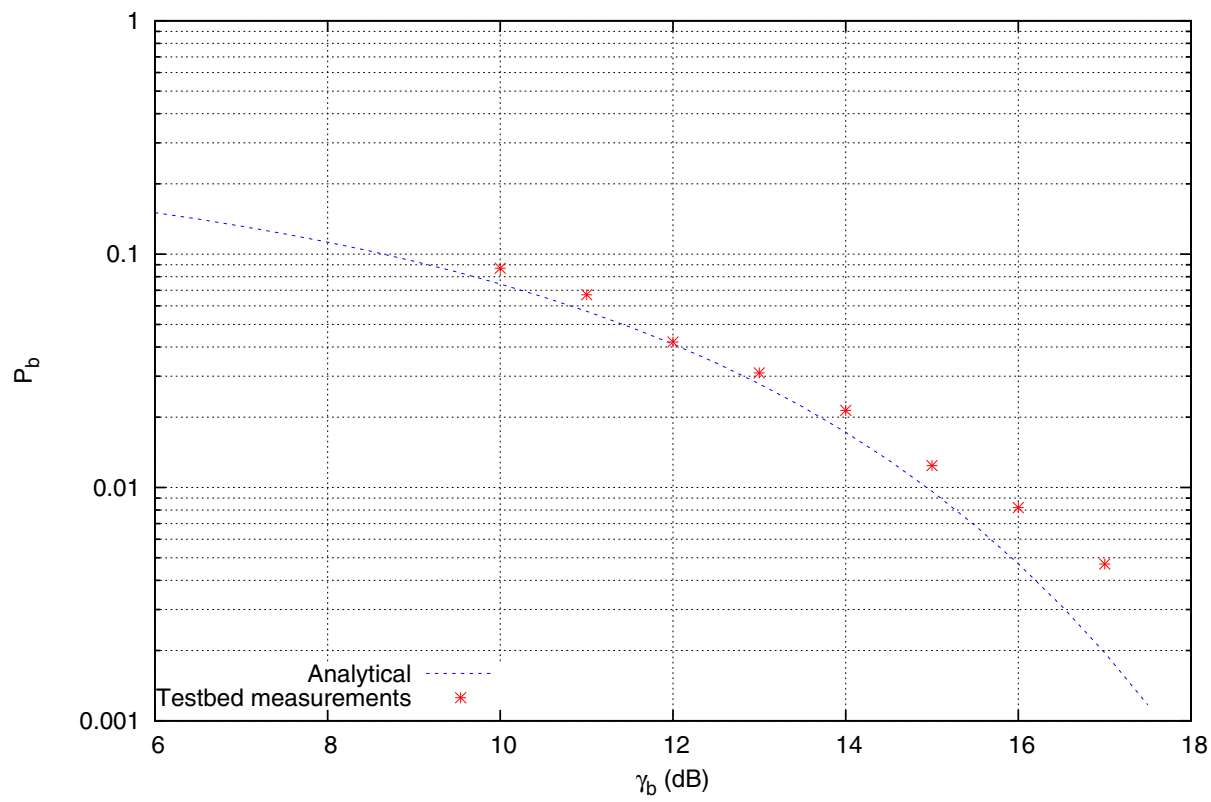

Figure 5 Bit error probability $\left(P_{b}\right)$ as a function of the SNR per bit $\left(\gamma_{b}\right)$ for the 3-Mbps rate mode (8-DPSK modulation). Comparison between the analytical approximation and the empirical measurements.

As a perfect synchronization is not achievable with actual hardware devices, the BER is under-estimated by the model, especially in scenarios with high SNR (and low BER). On the other hand, the practical impact of BER under 0.001 on the performance of BT is shown to be negligible. Thus, we consider that a particularized

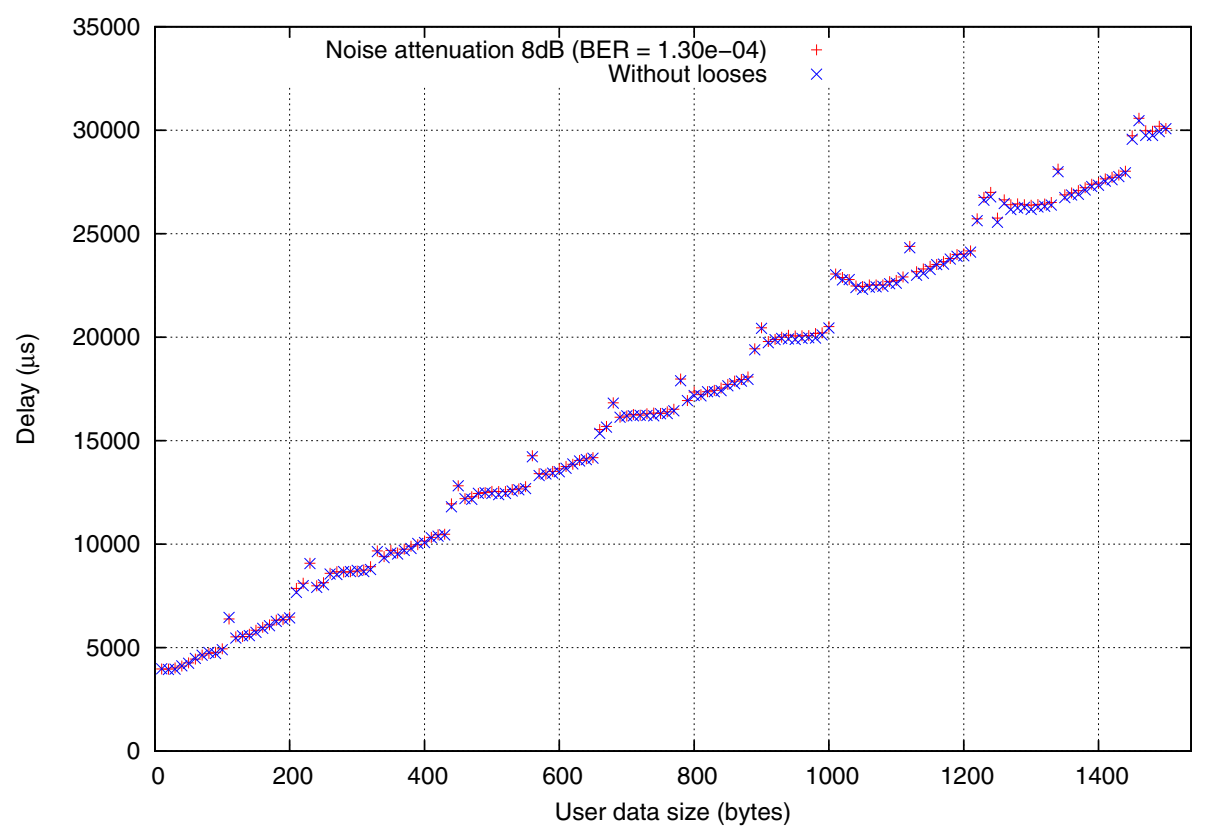

Figure 6 Transmission delay master-slave (DM packets), $T_{\text {poll }}=10 \mathrm{~ms}$. Comparison of the measured delay in a noise-free transmission and with a $8-\mathrm{dB}$ noise attenuation $(\mathrm{SNR}=14.95 \mathrm{~dB}$ ). Measurements obtained in the master-slave sense with DM packets and a poll interval of $10 \mathrm{~ms}$. 
model of the specific behavior of the employed modulator/demodulator is not required to evaluate the performance of BT in noisy environments.

\section{Conclusions}

Basing on existing analytical studies, the article has presented a model aimed at characterizing the BER of BT 2.x + EDR transmission. Following the common assumption of an AWGN channel, the presented formulas allow to calculate the BER as a function of the SNR. The formulas are particularized for the three different modulations (GFSK, $\pi / 4-D Q P S K$, and 8-DPSK) employed by BT 2.x+EDR.

The accuracy of the analytical model has been thoroughly evaluated in a real testbed implementing wired transmissions of commercial BT interfaces. The testbed included two signal attenuators and a calibrated generator of white noise, which is added to the BT signal through a combiner. By regulating both the transmission power of the emitting devices (which has been characterized in a spectrum analyzer) and the noise level at the noise generator, different noise conditions in the channel were emulated. For the different values of the induced SNR, the BER of the actual transmissions is measured by the systematic emission of probe BT packets.

Measurements show that, for the three modulations, analytical models reasonably approximate the actual behavior of BT communications for BERs higher than $10^{-3}$. On the other hand, actual BER is underestimated as SNR increases most probably because models do not consider ISI introduced by the Gaussian filter. However, the analysis of the packet delay reveals that this inaccuracy of the model could be neglected for most practical purposes. This is due to the fact that, in scenarios with a BER lower that $10^{-3}$, packet delay remains almost unaffected with respect to the ideal case where no packet has to be retransmitted.

Consequently, the presented analytical model (based on the related literature) can be utilized to predict the feasibility of networking applications based on BT $2 . x+E D R$ as a function of the power of the environmental noise.

\begin{abstract}
Abbreviations
AWGN: additive white Gaussian noise; BER: bit error rate; BT: bluetooth; DPSK: differential phase shift keying; DQPSK: differential quaternary phase shift keying; EDR: enhanced data rates; FEC: forward error correction; FSK: frequency shift keying; GFSK: Gaussian frequency shift keying; LM: link manager; LS: least squares; ML: maximum likelihood; SNR: signal-to-noise ratio; SPI: serial peripheral interface.
\end{abstract}

Acknowledgements

This study was supported by the Project No. TEC2009-13763-C02-01.

\section{Competing interests}

The authors declare that they have no competing interests.

Received: 17 September 2011 Accepted: 8 March 2012

Published: 8 March 2012
References

1. Bluetooth SIG, Bluetooth Core Specification Version 2.0+EDR, In Specification of the Bluetooth System. Bluetooth Special Interest Group. (10 November 2004). https://www.bluetooth.org/Technical/Specifications/adopted.htm

2. Bluetooth $\mathrm{SIG}$, Bluetooth Core Specification Version $2.1+\mathrm{EDR}$, In Specification of the Bluetooth System. Bluetooth Special Interest Group. (26 July 2007). https://www.bluetooth.org/Technical/Specifications/adopted.htm

3. M Shimizu, N Aoki, K Shirakawa, Y Tozawa, N Okubo, Y Daido, New method of analyzing BER performance of GFSK with postdetection filtering. IEEE Trans Commun. 45(4), 429-436 (1997). doi:10.1109/26.585918

4. A Chandra, SC Mandal, C Bose, BER of $\pi / 4-D Q P S K$ with multichannel reception: some series solutions, in TENCON 2008 - 2008 IEEE Region 10 Conference, Hyderabad (India), 1-6 (November, 2008)

5. I Al Falujah, VK Prabhu, Error performance of DQPSK with EGC diversity reception over fading channels. IEEE Trans Wirel Commun. 7(4), 1190-1194 (2008)

6. K Kiasaleh, T He, Performance of DQPSK communication systems impaired by mixer imbalance, timing error, and Rayleigh fading, in ICC 96, IEEE International Conference on Communications, 1996, Dallas (TX, USA), 1, 364-368 (1996)

7. K Kiasaleh, T He, On the performance of DQPSK communication systems impaired by timing error, mixer imbalance, and frequency nonselective slow Rayleigh fading. IEEE Trans Veh Technol. 46(3), 642-652 (1997). doi:10.1109/25.618190

8. LE Miller, JS Lee, BER expressions for differentially detected $\pi / 4-D Q P S K$ modulation. IEEE Trans Commun. 46(1), 71-81 (1998). doi:10.1109/26.655405

9. CS Ng, TT Tjhung, F Adachi, KM Lye, On the error rates of differentially detected narrowband $\pi / 4-D Q P S K$ in Rayleigh fading and Gaussian noise IEEE Trans Veh Technol. 42(3), 259-265 (1993). doi:10.1109/25.231876

10. Y Sun, A Baricz, M Zhao, X Xu, S Zhou, Approximate average bit error probability for DQPSK over fading channels. Electron Lett. 45(23), 1177-1179 (2009). doi:10.1049/el.2009.2467

11. DPC Wong, PT Mathiopoulos, Nonredundant error correction analysis and evaluation of differentially detected $\pi / 4$-shift DQPSK systems in a combined CCI and AWGN environment. IEEE Trans Veh Technol. 41(1), 35-48 (1992). doi:10.1109/25.120143

12. YC Chow, AR Nix, JP McGeehan, An error bound analysis for M-DPSK in frequency-selective Rayleigh fading channel with diversity reception, in Singapore ICCS '94 Conference Proceedings, Singapore, 2, 686-690 (1994)

13. I Kostic, Average symbol error probability for M-ary DDPSK. Electron Lett. 44(20), 1203-1204 (2008). doi:10.1049/el:20081934

14. DL Noneaker, MB Pursley, Error probability bounds for M-PSK and M-DPSK and selective fading diversity channels. IEEE Trans Veh Technol. 43(4), 997-1005 (1994). doi:10.1109/25.330162

15. H Liu, V Venkatesan, C Nilsen, R Kyker, ME Magana, Performance of frequency hopped noncoherent GFSK in correlated Rayleigh fading channels, in ICC '03, IEEE International Conference on Communications, 2003, Anchorage (AK, USA), 4, 2779-2783 (2003)

16. C Tibenderana, S Weiss, Low-complexity high-performance GFSK receiver with carrier frequency offset correction, in IEEE Proceedings of International Conference on Acoustics, Speech, and Signal Processing, 2004 (ICASSP'04), Montreal (Canada), 4, 933-936 (2004)

17. A García-Armada, BB Rodríguez, VPG Jiménez, M Sánchez-Fernández, Modelling, performance analysis and design of WPAN systems. Wirel Personal Commun. 42(3), 367-386 (2007). doi:10.1007/s11277-006-9183-x

18. DC Chang, TH Shiu, Digital GFSK carrier synchronization, in APCCAS 2006, IEEE Asia Pacific Conference on Circuits and Systems, 2006, Singapore, 1523-1526 (2006)

19. DC Chang, Least squares/maximum likelihood methods for the decisionaided GFSK receiver. IEEE Signal Process Lett. 16(6), 517-520 (2009)

20. MC Valenti, M Robert, JH Reed, On the throughput of Bluetooth data transmissions, in WCNC2002, IEEE Wireless Communications and Networking Conference, 2002, Orlando (FL, USA), 1, 119-123 (2002)

21. JP Skudlarek, RadiSim-a fast digital RF behavioral simulator including bit error rate assessment for system exploration, validation, and tuning, in BMAS 2002, Proceedings of the 2002 IEEE International Workshop on Behavioral Modeling and Simulation, 2002, Santa Rosa (CA, USA), 61-66 (6-8 October, 2002)

22. JH Yoon, SB Lee, SC Park, Packet and modulation type selection scheme based on channel quality estimation for Bluetooth evolution systems, in WCNC 2004, IEEE Wireless Communications and Networking Conference, Atlanta (GA, USA), 2, 1014-1017 (March, 2004) 
23. J Sung Roh, Performance analysis and evaluation of bluetooth networks in wireless channel environment, in ICSNC'06, International Conference on Systems and Networks Communications, 2006, Tahiti, 61 (October, 2006)

24. Y Hua, Y Zou, Analysis of the packet transferring in L2CAP layer of Bluetooth v2.x+EDR, in International Conference on Information and Automation, 2008. ICIA 2008, Zhangjiajie, China, 753-758 (2008)

25. J Mikulka, S Hanus, Bluetooth EDR physical layer modeling, in 18th International Conference Radioelektronika, 2008, Prague, 1-4 (24-25 April, 2008)

26. IY Moon, Performance analysis of WAP in bluetooth ad-hoc network system, in Knowledge-Based Intelligent Information and Engineering Systems. Lecture Notes in Computer Science, vol. 4692/2010, Springer-Verlag, Heidelberg, 390-396 (2007)

27. A Zanella, A mathematical framework for the performance analysis of bluetooth with enhanced data rate. IEEE Trans Commun. 57(8), 2463-2473 (2009)

28. A Zanella, Analysis of the packet reception statistics of Bluetooth $\mathrm{V} 2+\mathrm{EDR}$ in fading channels, Technical Report 155, Dep. of Information Engineering, University of Padova, Italy, (2007)

29. A Goldsmith, Wireless Communications (Cambridge University Press, Cambridge, 2005)

30. A Bruce Carlson, Communication Systems: An Introduction to Signals and Noise in Electrical Communication, McGraw-Hill Education, New York, 3rd edn. (1986)

31. J Proakis, Digital Communications, 4th edn. (McGraw-Hill Science/ Engineering/Math, New York, 2001)

32. MK Simon, MS Alouini, Digital Communication over Fading Channels. Wiley Series in Telecommunications and Signal Processing, 2nd edn. (Wiley-IEEE Press, Hoboken, NJ, USA, 2005)

33. BlueGiga Tech. Bluegiga Technologieshttp://www.bluegiga.com. Accessed 16 June 2010

34. BlueGiga Tech. WT11 Evaluation Kithttp://www.bluegiga.com/eval_wt11. Accessed 16 June 2010

35. Agilent Tech. 8494B Manual Step Attenuator, DC to $18 \mathrm{GHz}, 0$ to $11 \mathrm{~dB}, 1$ dB steps http://www.home.agilent.com/agilent/product.jspx?pn=8496B. Accessed 21 June 2010

36. Agilent Tech. 8496B Manual Step Attenuator, DC to $18 \mathrm{GHz}, 0$ to $110 \mathrm{~dB}, 10$ dB steps http://www.home.agilent.com/agilent/product.jspx?pn=8496B. Accessed 21 June 2010

37. NoiseCom. Analog Noise Generators NC6000A/8000A http://noisecom.com/ products/instruments/nc6000a-8000a-series. Accessed 21 July 2010

38. ITU-T, ITU-T Recommendation 0.150, International Telecommunication Union, (1996)

39. Agilent Tech. N9010A EXA Signal Analyzer http://www.home.agilent.com/ agilent/product.jspx?cc=GB\&lc=eng\&ckey=1160741\&nid=-33932.710350. 00\&id=1160741\&cmpid=28198. Accessed 21 June 2010

40. D Han, Y Zheng, An ultra low power GFSK demodulator for wireless body area network, in ESSCIRC 2008, 34th European Solid-State Circuits Conference, 2008, Edinburg (UK), 434-437 (September, 2008)

41. UD Han, Y Zheng, A GFSK demodulator based on instant phase computation and adaptive multi-threshold quantization, in A-SSCC 2009, IEEE Asian Solid-State Circuits Conference 2009, Singapore, 249-252 (16-18 November, 2009)

42. N Ibrahim, L Lampe, R Schober, Bluetooth receiver design based on Laurent's decomposition. IEEE Trans Veh Technol. 56(4), 1856-1862 (2007)

43. HS Kao, MJ Yang, TC Lee, A delay-line-based GFSK demodulator for low-IF receivers, in ISSCC 2007, 54th IEEE International Solid-State Circuits Conference, San Francisco (CA, USA), (11-15 February, 2007)

44. TC Lee, CC Chen, A mixed-signal GFSK demodulator for Bluetooth. IEEE Trans Circ Syst II: Exp Briefs. 53(3), 197-201 (2006)

45. B Xia, C Xin, W Sheng, AY Valero-Lopez, E Sánchez-Sinencio, A GFSK demodulator for low-IF Bluetooth receiver. IEEE J Solid State Circ. 38(8), 1397-1400 (2003). doi:10.1109/JSSC.2003.814424

46. C Xin, B Xia, W Sheng, AY Valero-Lopez, E Sanchez-Sinencio, A mixed-mode IF GFSK demodulator for Bluetooth, in ISCAS 2002, IEEE International Symposium on Circuits and Systems, 2002, Scottsdale (AZ, USA), 3, 457-460 (2002)

47. W Sheng, B Xia, AE Emira, C Xin, AY Valero-López, ST Moon, E SánchezSinencio, A 3-V, 0.35- $\mathrm{mm}$ CMOS Bluetooth receiver IC. IEEE J Solid State Circ. 38(1), 30-42 (2003). doi:10.1109/JSSC.2002.806277
48. SB Hyun, GY Tak, SH Kim, BJ Kim, J Ko, SS Park, A dual-mode 2.4-Ghz CMOS transceiver for high-rate Bluetooth systems. ETRI J. 26(3), 229-240 (2004). doi:10.4218/etrij.04.0103.0090

doi:10.1186/1687-1499-2012-94

Cite this article as: Luque et al: Analytical and empirical evaluation of the impact of Gaussian noise on the modulations employed by Bluetooth Enhanced Data Rates. EURASIP Journal on Wireless Communications and Networking 2012 2012:94.

\section{Submit your manuscript to a SpringerOpen ${ }^{\odot}$ journal and benefit from:}

- Convenient online submission

- Rigorous peer review

- Immediate publication on acceptance

- Open access: articles freely available online

- High visibility within the field

- Retaining the copyright to your article

Submit your next manuscript at $>$ springeropen.com 